Article

\title{
Optically Driven Mobile Integrated Micro-Tools for a Lab-on-a-Chip
}

\author{
Yi-Jui Liu ${ }^{1}$, Yi-Hsiung Lee ${ }^{2}$, Yu-Sheng Lin ${ }^{3}$, Chingfu Tsou ${ }^{1}$, Patrice L. Baldeck ${ }^{3,4}$ and \\ Chih-Lang Lin ${ }^{3, *}$
}

1 Department of Automatic Control Engineering, Feng Chia University, Taichung 407, Taiwan; E-Mails: erliu@fcu.edu.tw (Y.-J.L.); cftsou@fcu.edu.tw (C.T.)

2 Ph.D. Program of Electrical and Communications Engineering, Feng Chia University, Taichung 407, Taiwan; E-Mail: andy955305@gmail.com

3 Institute of Biomedical Engineering and Materials Science, Central Taiwan University of Science and Technology, Taichung 406, Taiwan; E-Mails: m09905009@ms3.ctust.edu.tw (Y.-S.L.); baldeck@ujf-grenoble.fr (P.L.B.)

4 University Grenoble, I/CNRS, LIPhy UMR 5588, Grenoble, F-38041, France

* Author to whom correspondence should be addressed; E-Mail: cllin101943@ctust.edu.tw; Tel.: +886-4-2239-1647 (ext. 6803); Fax: +886-4-2239-4256.

Received: 2 February 2013; in revised form: 18 March 2013 / Accepted: 29 March 2013 / Published: 11 April 2013

\begin{abstract}
This study proposes an optically driven complex micromachine with an Archimedes microscrew as the mechanical power, a sphere as a coupler, and three knives as the mechanical tools. The micromachine is fabricated by two-photon polymerization and is portably driven by optical tweezers. Because the microscrew can be optically trapped and rotates spontaneously, it provides driving power for the complex micro-tools. In other words, when a laser beam focuses on the micromachine, the microscrew is trapped toward the focus point and simultaneously rotates. A demonstration showed that the integrated micromachines are grasped by the optical tweezers and rotated by the Archimedes screw. The rotation efficiencies of the microrotors with and without knives are $1.9 \mathrm{rpm} / \mathrm{mW}$ and $13.5 \mathrm{rpm} / \mathrm{mW}$, respectively. The micromachine can also be portably dragged along planed routes. Such Archimedes screw-based optically driven complex mechanical micro-tools enable rotation similar to moving machines or mixers, which could contribute to applications for a biological microfluidic chip or a lab-on-a-chip.
\end{abstract}


Keywords: archimedes screw; microrotor; optical tweezers; two-photon polymerization

\section{Introduction}

The lab-on-a-chip has attracted scientific interest from related biological fields because it is convenient, rapid, and accurate, while requiring few consumables. Many studies [1-6] have examined the technology. Specially designed micromachines with critical components increase lab-on-a-chip functions. In this case, optically driven micromachines are important because they have no mechanical contact, no electrical wires, are controlled by a remote, and enable variable manipulation. These optically driven tools perform mechanical and sensing functions on a micro and nanoscale. Using this technique, several optically driven elemental micromachines have been proposed, such as micropumps [7-10], microactuators [11,12], and microvalves [13]. They are expected to contribute to manipulating, sorting, drilling, and treating biological cells. Recent studies have focused on more complex machines that integrate the basic elements with additional mechanisms for performing multiplicate operations [14-18], and the issue of optical driving on asymmetrical objects [19]. In this case, a transmission with precise applied force control is essential for operating the complex micromachines. Moreover, to fulfill variable functional biological application requirements, microfluidic chip operation is becoming more complex and diverse. However, few studies have focused on the relationship between driving power and functional tools, and most mechanisms in these studies are immobile in the microchannels.

In this study, we fabricated Archimedes screw-based micromachines using two-photon polymerization (TPP). As in the previous works [20,21], the Archimedes screw is optically trapped and rotates spontaneously. This function provides the power source for the integrated complex micro-tools. Finally, an optically driven mobile micromachine is also tested.

\section{Fabrication of Archimedes Screw-Based Micromachines}

TPP is a convenient technique for fabricating 3D microstructures with arbitrary 3D shapes [22]. Structures with complex shapes can be directly obtained by scanning a laser-focusing point along predetermined trajectories. This study fabricates micromachines using a commercial TPP 3D-microfabrication machine (Teem Photonics Inc.) with a passively Q-switched Nd:YAG microchip laser with a $532 \mathrm{~nm}$ wavelength. The laser beam was expanded by a telescope coupled to an inverted microscope (Olympus IX51) and focused using a microscope objective lens (100×, NA $=1.3$ ). Commercial resin (Photomer 3015, Henkel Inc., Ambler, PA, USA) was used with a photo-initiator specifically designed for two-photon absorption. Polymerization occurred at the focal point in the resin with less than $0.1 \mathrm{~mW}$ of laser power and a $1 \mathrm{~ms}$ exposure. Figure 1 shows a diagram of the experiment.

To demonstrate optical manipulation, a three-blade Archimedes screw with a 1/3 cycle was fabricated. The screw was $4 \mu \mathrm{m}$ in diameter and $10 \mu \mathrm{m}$ high. The sphere and complex microrotor were $3 \mu \mathrm{m}$ and $13 \mu \mathrm{m}$ in diameter, respectively. Figure 2 shows the dimensions of the Archimedes screw-based micromachines. A SEM photograph of the TPP microfabrication product is shown in Figure 3. 
Figure 1. The two-photo polymerization 3-D microfabrication system.

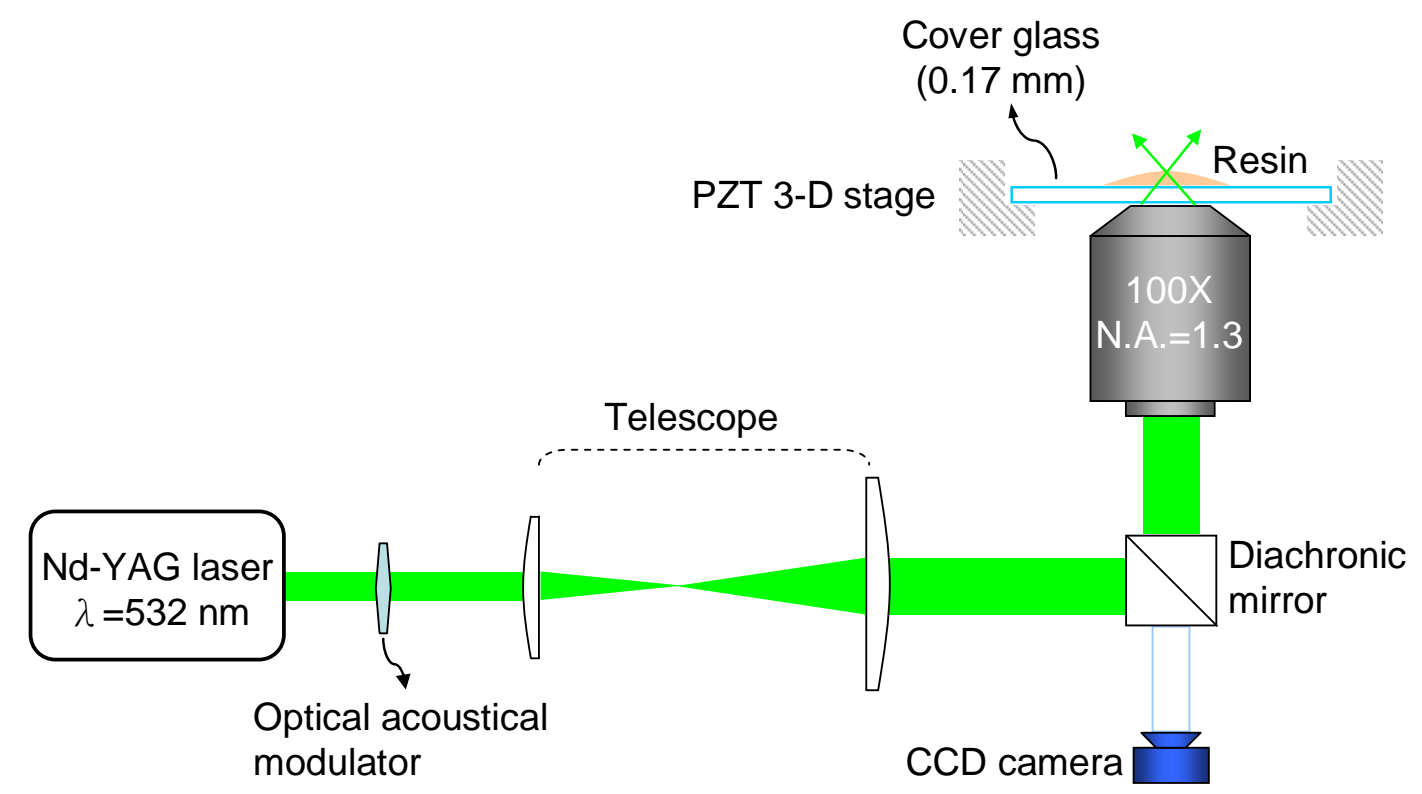

Figure 2. Dimension of the Archimedes screw-based micromachines: (a) 3-D view; (b) side view; (c) bottom view.

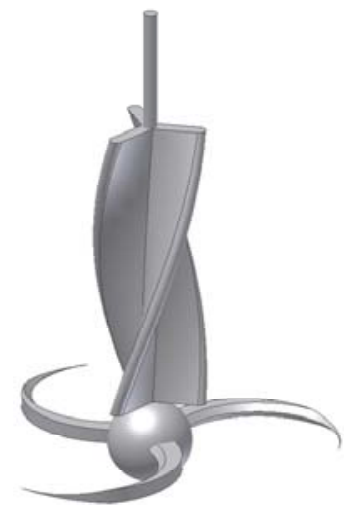

(a)

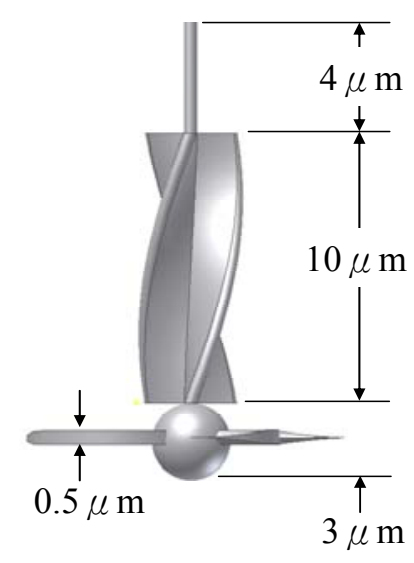

(b)

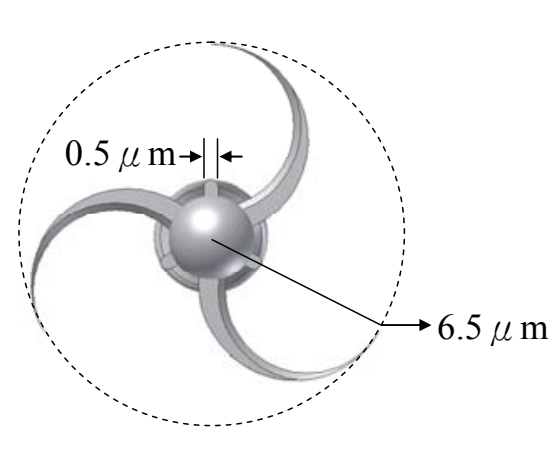

(c)

Figure 3. The SEM figures (top-view) of the Archimedes screw-based micromachines (left) and Archimedes-screws (right).
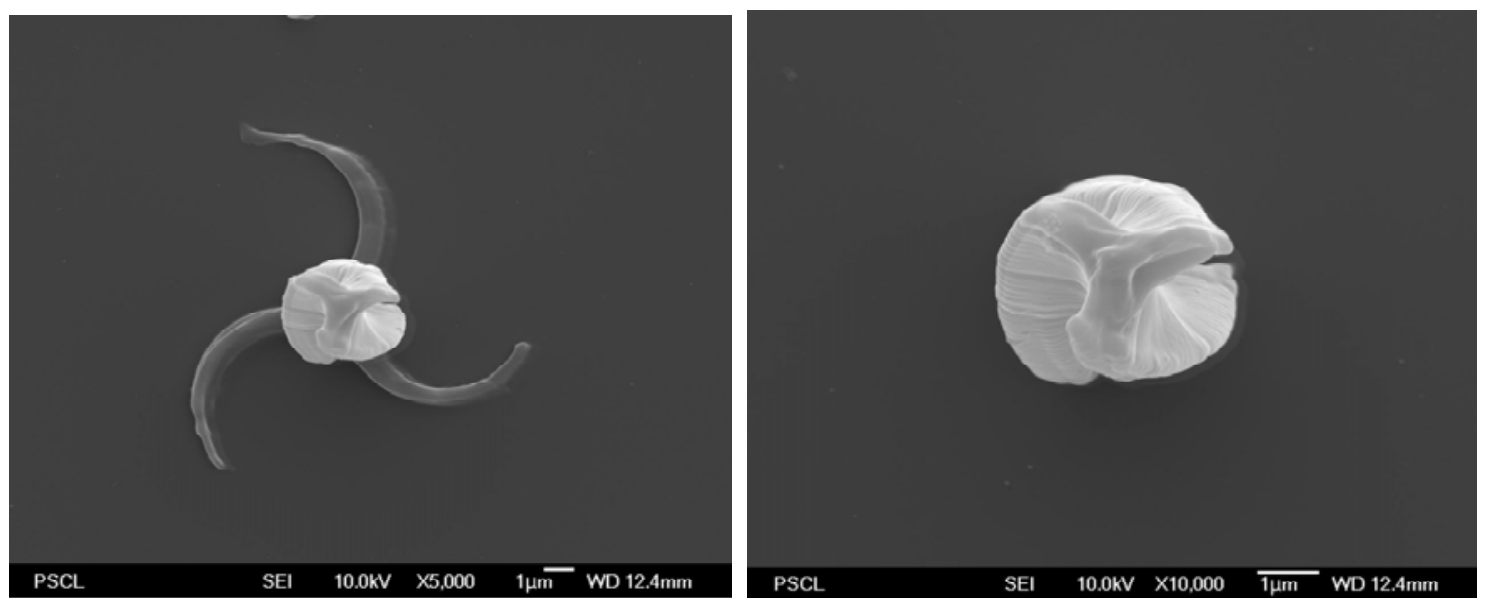


\section{Optical Driving Demonstration}

A commercial optical tweezers (PALM MicroTweezers, ZEISS Inc.) is used to perform the optical driving demonstration. The micro-tools are grasped and rotated by the Archimedes screw, as shown in Figure 4. Because elongated objects in optical traps align along the beam axis [23], a rod was designed at the center of the Archimedes screw. The height and diameter of the integrated micro-tool were $17 \mu \mathrm{m}$ and $13 \mu \mathrm{m}$, respectively, ensuring that the structure aligns along the laser beam axis. In the earlier tests, the knives were directly combined with the blades of the Archimedes screw. The result showed that the integrated micro-tool could not rotate symmetrically, and that it was not stable when dragged by the optical tweezers. In this study, a sphere was set as a coupler below the screw to connect the three knives. It also acted as an anchor to stabilize the micro-tool.

Figure 4. Schematic figures of the optically driven integrated micro-tool: once the optical tweezers turns on, the micro-tool is trapped toward the laser focus, and then the Archimedes screw is spontaneously rotated by the optical tweezers, and drives the micro-tool.

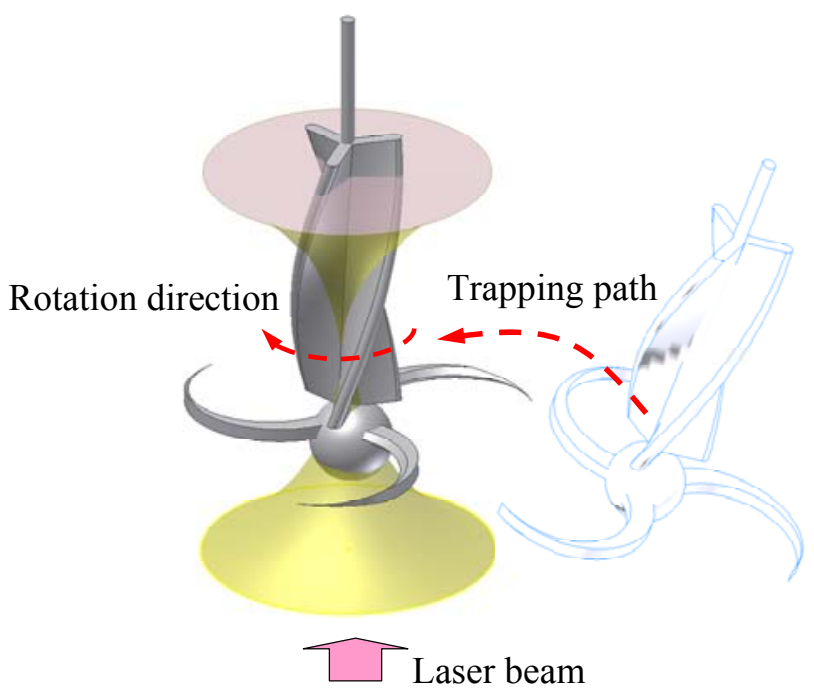

The rotational speeds of the micromachines were measured by detecting the light period perpendicular to the image plane of the knives. An oscilloscope was used to visualize the photodiode signal. The laser powers were measured at the objective. Figure 5 shows the demonstration results. The rotational efficiencies of the Archimedes screw and the integrated micro-tool (screw and knives) were $10.3 \mathrm{rpm} / \mathrm{mW}$ and $1.8 \mathrm{rpm} / \mathrm{mW}$, respectively. The optical driving mobile micro-tool was demonstrated with $80 \mathrm{~mW}$ of laser power, as shown in Figure 6 . The optical tweezers dragged the micro-tool along the planed routes in an S-shape and a corner shape. The results indicate that the micro-tool is easily carried anywhere along variable routes. 
Figure 5. The rotational efficiencies $(\mathrm{rpm} / \mathrm{mW})$ of the Archimedes screw and the integrated micro-tool (screw + knives).

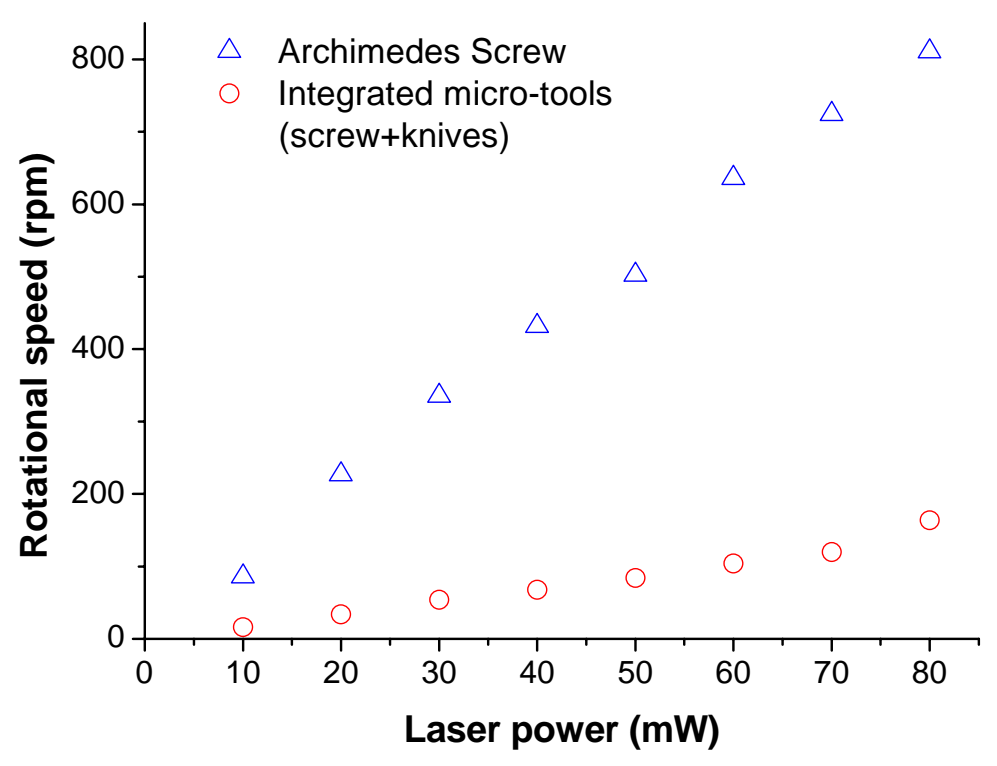

Figure 6. The demonstrations of the optical driving mobile micro-tools: (a) an S-shape; (b) a corner shape.
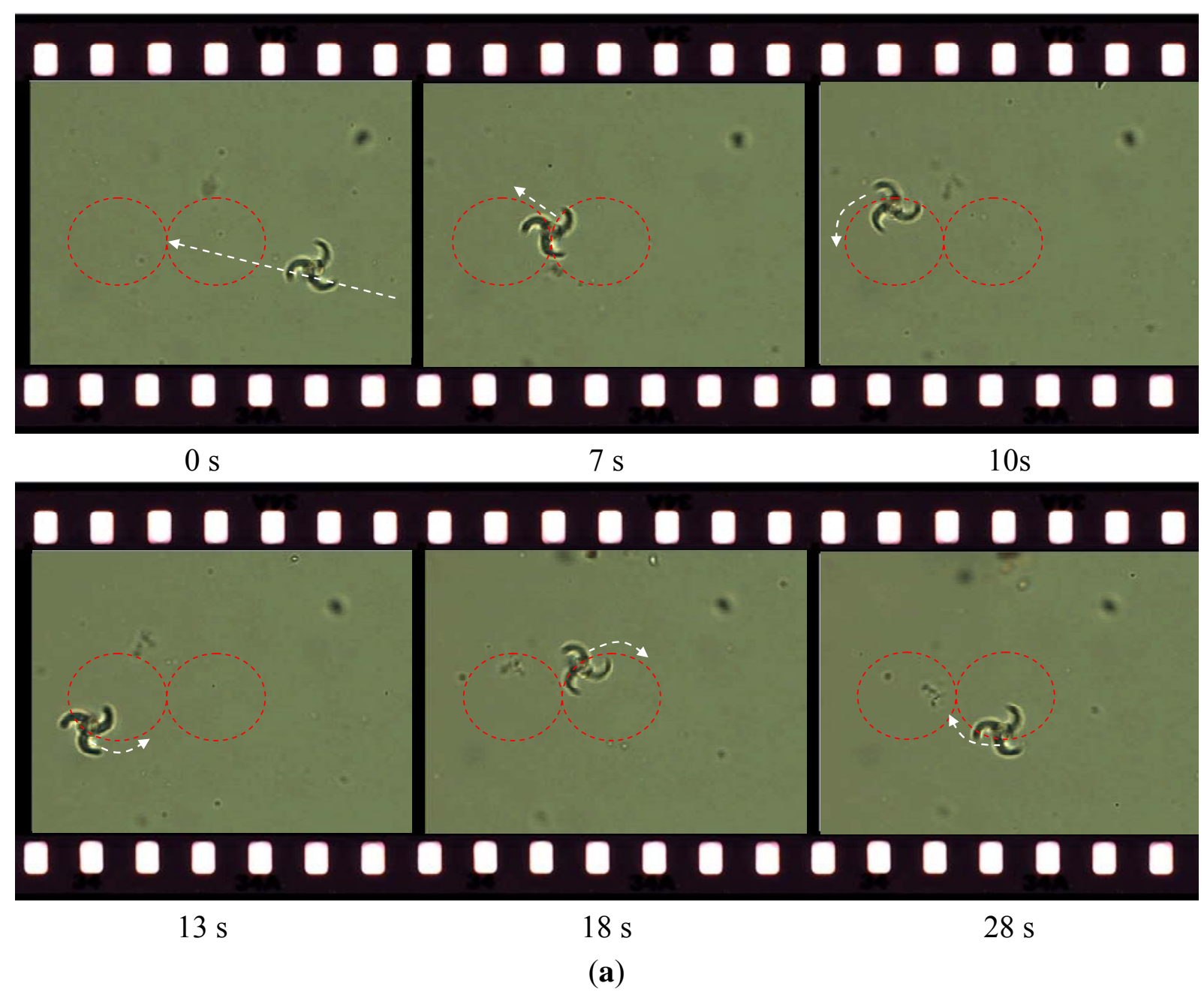
Figure 6. Cont.
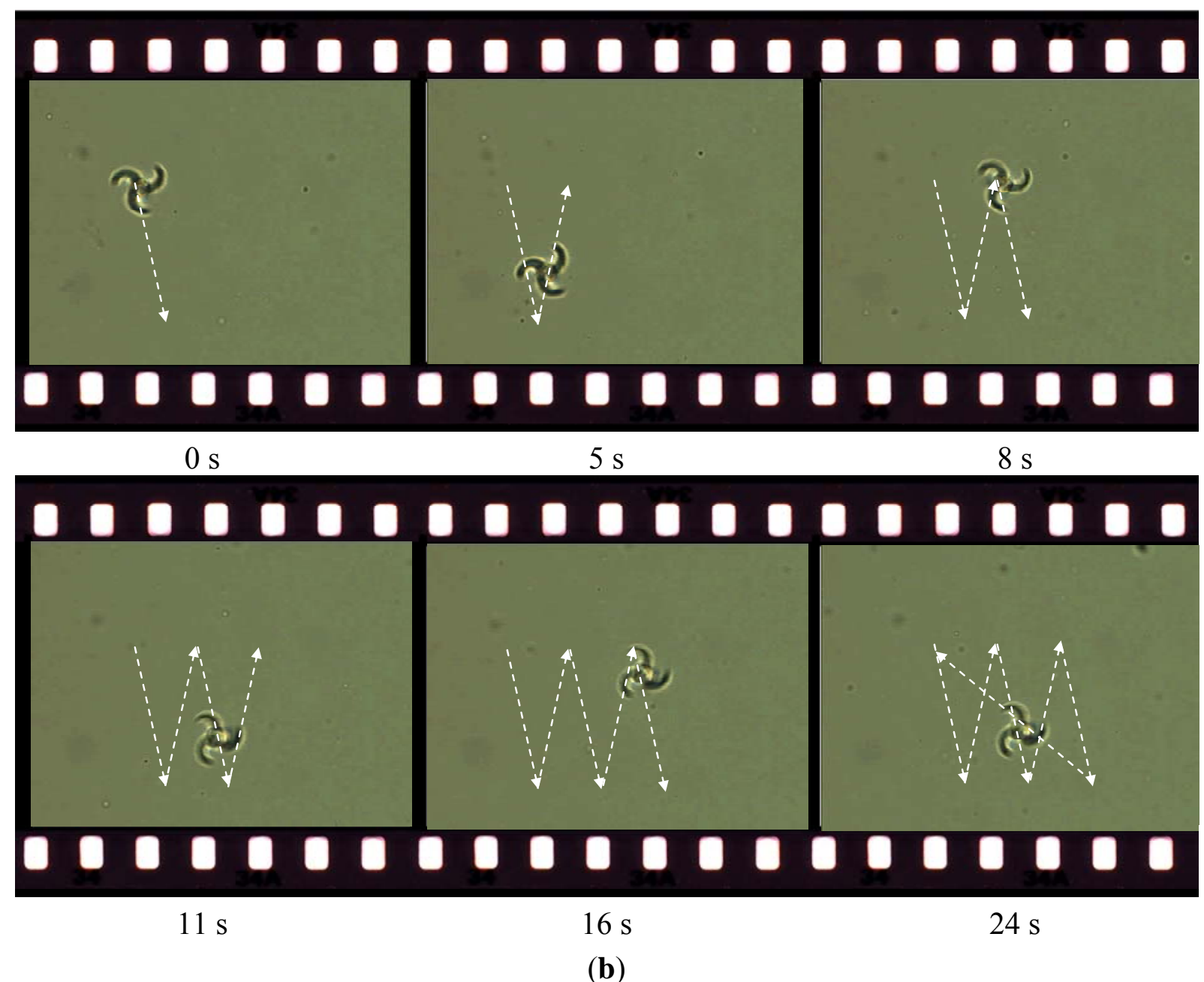

\section{Conclusions}

This study successfully fabricated a complex optically driven micro-tool using TPP. The micro-tool was integrated with three components: an Archimedes screw as the driving power to transmit the optical force, three knives as functional tools, and a sphere as the coupler to connect the screw and knives. Such optically controlled micromachines provide a new method of directly transmitting applied optical forces to functional tools. This optically driven mechanism is expected to be a versatile, powerful tool for mobile manipulation in microfluidic channel and lab-on-a-chip applications.

\section{Acknowledgments}

This work was funded by the National Science Council (NSC 101-2221-E-166-003-).

\section{References}

1. Baldeck, P.L.; Lin, C.L.; Andraud, C. Two-photon absorption of organics: From spectroscopy to photodriven microsensors. Stud. Univ. Cluj-Napoca Ser. Phys. 2004, 2, 75-79.

2. Lin, C.-L.; Wang, I.; Dollet, B.; Baldeck, P.L. Velocimetry microsensors driven by linearly polarized optical tweezers. Opt. Lett. 2006, 31, 329-331. 
3. Eriksson, E.; Enger, J.; Nordlander, B.; Erjavec, N.; Ramser, K.; Goksör, M.; Hohmann, S.; Nyström, T.; Hanstorp, D. A microfluidic system in combination with optical tweezers for analyzing rapid and reversible cytological alterations in single cells upon environmental changes. Lab Chip 2007, 7, 71-76.

4. Boer, G.; Johann, R.; Rohner, J.; Merenda, F.; Delacrétaz, G.; Renaud, P.; Salathé, R.-P. Combining multiple optical trapping with microflow manipulation for the rapid bioanalytics on microparticles in a chips. Rev. Sci. Instrum. 2007, 78, 116101:1-116101:3.

5. Lin, C.-C.; Chen, A.; Lin, C.-H. Microfluidic cell counter-sorter utilizing multiple particle tracing technique and optically switching approach. Biomed. Microdevices 2008, 10, 56-63.

6. Mohanty, S. Optically-actuated translational and rotational motion at the microscale for microfluidic manipulation and characterization. Lab Chip 2012, 12, 3624-3636.

7. Lin, C.-L.; Wang, I.; Pierre, M.; Colombier, I.; Andraud, C.; Baldeck, P.L. Rotational properties of micro-slabs driven by linearly polarized light. J. Nonlinear Opt. Phys. Mater. 2005, 14, 375-382.

8. Whyte, G.; Gibson, G.; Leach, J.; Padgett, M. Rotation of micron-sized objects. Opt. Express 2006, 14, 12497-12502.

9. Kelemen, L.; Valkai, S.; Ormos, P. Integrated optical motor. Appl. Opt. 2006, 45, 2777-2780.

10. Maruo, S.; Inoue, H. Optically driven micropump produced by three-dimensional two-photon microfabrication. Appl. Phys. Lett. 2006, 89, 144101-144103.

11. Lin, C.-L.; Lee, Y.-H.; Lin, C.-T.; Liu, Y.-J.; Hwang, J.-L.; Chung, T.-T.; Baldeck, P.L. Multiplying optical tweezers force using a micro-lever. Opt. Express 2011, 19, 20604-20609.

12. Lin, C.-L.; Li, Y.-H.; Lin, C.-T.; Chiang, C.-C.; Liu, Y.-J.; Chung, T.-T.; Baldeck, P.L. Preliminary Study of Lever-based Optically Driven Micro-actuator. In Proceedings of the 3rd International Conference on Smart Materials and Nanotechnology in Engineering, ShenZhen, China, 5-8 Decmber 2011.

13. Lin, C.-L.; Huang, C.; Tseng, C.-L.; Baldeck, P.L.; Chung, T.-T. Fabrication of Micro-Malves by Two-Photon Polymerization for Microfluidics Applications. In Proceedings of The 5th International Conference on Nanophotonics, Shanghai, China, 22-26 May 2011.

14. Maruo, S.; Hiratsuka, Y. Optically driven micromanipulators with rotating arms. J. Rob. Mechatron. 2007, 19, 565-568.

15. Maruo, S.; Takaura, A.; Saito, Y. Optically driven micropump with a twin spiral microrotor. Opt. Express 2009, 17, 18525-18532.

16. Ito, K.; Frusawa, H.; Kimura, M. Precise switching control of liquid crystalline microgears driven by circularly polarized light. Opt. Express 2012, 20, 4254-4259.

17. Dong, X.-Z.; Zhao, Z.-S.; Duan, X.-M. Micronanofabrication of assembled three-dimensional microstructures by designable multiple beams multiphoton processing. Appl. Phys. Lett. 2007, 91, 124103: 1-124103:3.

18. Sun, S. Fabrication technology of involute micro gear based on two-photon of femtosecond laser. Appl. Mech. Mater. 2011, 44, 670-674.

19. Glückstad, J. Optical manipulation: Sculpting the object. Nat. Photonics 2011, 5, 7-8.

20. Lin, C.-L.; Vitrant, G.; Bouriau, M.; Casalegno, R.; Baldeck, P.L. Optically driven archimedes micro-screws for micropump applications. Opt. Express 2011, 19, 8267-8276. 
21. Baldeck, P.L.; Lin, C.-L.; Lin, Y.-S.; Lin, C.-T.; Chung, T.-T.; Bouriau, M.; Vitrantd, G. Optically driven archimedes micro-screws for micropump applications: Multiple blade design. Proc. SPIE 2011, doi: 10.1117/12.893679.

22. Maruo, S.; Nakamura, O.; Kawata, S. Three-dimensional microfabrication with two-photon-absorbed photopolymerization. Opt. Lett. 1997, 22, 132-134.

23. Nieminen, T.A.; Rubinsztein-Dunlop, H.; Heckenberg, N.R. Angular Momentum Generation by Scattering: Alignment Androtation of Microobjects. In Proceedings of 6th Conference on Electromagnetic and Light Scattering by Nonspherical Particles: Theory, Measurements,and Applications, Gainesville, FL, USA, 4-8 March 2002; pp. 239-242.

(C) 2013 by the authors; licensee MDPI, Basel, Switzerland. This article is an open access article distributed under the terms and conditions of the Creative Commons Attribution license (http://creativecommons.org/licenses/by/3.0/). 